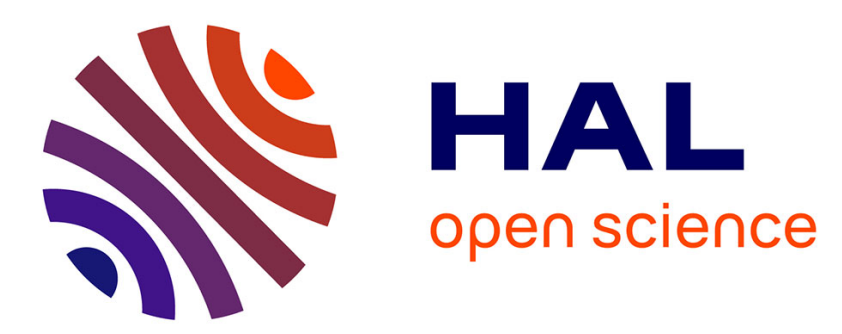

\title{
Infrastructures publiques et financement privé: le paradoxe de la rentabilité financière
}

\author{
Alain Bonnafous
}

\section{To cite this version:}

Alain Bonnafous. Infrastructures publiques et financement privé: le paradoxe de la rentabilité financière. Revue d'économie financière, 1999, Le financement des infrastructures, 51, pp.157-166. halshs-00325170

\section{HAL Id: halshs-00325170 \\ https://shs.hal.science/halshs-00325170}

Submitted on 2 Sep 2016

HAL is a multi-disciplinary open access archive for the deposit and dissemination of scientific research documents, whether they are published or not. The documents may come from teaching and research institutions in France or abroad, or from public or private research centers.
L'archive ouverte pluridisciplinaire HAL, est destinée au dépôt et à la diffusion de documents scientifiques de niveau recherche, publiés ou non, émanant des établissements d'enseignement et de recherche français ou étrangers, des laboratoires publics ou privés. 


\title{
INFRASTRUCTURES PUBLIQUES ET FINANCEMENT PRIVÉ : LE PARADOXE DE LA RENTABILITÉ FINANCIÈRE
}

\author{
AlAIN BONNAFOUS*
}

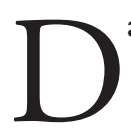
a décision d'investir dans un projet d'infrastructure est, certes, la plus spectaculaire des mesures de politique des transports, ne serait-ce qu'en raison des charmes des inaugurations (ou des manifestations de riverains). Pour autant, elle n'est que l'un des instruments de cette politique ; un instrument qui doit être utilisé en cohérence avec d'autres : doit-on décider d'investir sans qu'un financement soit assuré, sans qu'un principe de tarification d'usage de l'infrastructure soit établi ou encore sans que l'efficacité économique du projet soit évaluée et prise en compte? Raisonnablement on ne le doit pas ${ }^{1}$. Or le mode de financement, le dispositif de tarification ou les critères d'évaluation sont bien, également, des manettes de la politique de transport qui forment, avec la décision d'investir, une sorte de carré magique. Un carré d'options ${ }^{2}$ qui doivent assurer le développement des réseaux selon le meilleur rendement social possible des ressources qui leur sont affectées. Un carré magique, car elles doivent l'assurer ensemble, ce qui est moins simple que ne le suggèrent les prescriptions de la théorie économique.

C'est ce défi que nous nous proposons d'explorer, en commençant par esquisser les difficultés qui apparaissent lorsque l'on passe d'une logique d'investissement d'une entreprise privée à un investissement qui s'inscrit dans une raison économique d'Etat.

\section{DU PRIVÉ AU PUBLIC}

Le carré est, en somme, moins magique lorsque l'on se place dans la seule logique marchande, comme celle qui préside à l'investissement industriel ou comme celle qui a alimenté le développement de notre réseau ferré, au XIX ${ }^{\text {ème }}$ siècle, dans sa phase la plus dynamique. Etablie

\footnotetext{
* Professeur de sciences économiques, Laboratoire d'Economie des Transports
} 
sur la base des prix qui sont imposés par le marché et d'une anticipation de la demande, la perspective de profit détermine la décision d'investir.

Lorsque l'entrepreneur dispose d'une capacité de financement, l'évaluation consiste en une confrontation de la rentabilité propre au projet considéré à celle de placements alternatifs, par exemple sur le marché financier. Lorsqu'il ne peut financer que par l'emprunt, c'est au coût de cet emprunt sur ce même marché qu'est comparée la rentabilité du projet. Celle-ci est mesurée par le taux de rentabilité interne (TRI) qui est défini comme le taux d'actualisation pour lequel le solde du bilan actualisé du projet, ou valeur actualisée nette (VAN), est égal à zéro. Il s'agit, pour l'entrepreneur, d'un bilan des coûts et des recettes tels qu'ils peuvent être prévus dans la durée couverte par le bilan, ce qui implique une marge de sécurité liée aux incertitudes de ces prévisions.

Ainsi, dans les circonstances actuelles, une grande entreprise industrielle qui peut obtenir un financement de long terme à un taux réel ${ }^{3}$ de l'ordre de $4 \%$, établit aux environs de $12 \%$ le TRI à partir duquel elle décide d'investir. Cet écart, de l'ordre de huit points, est réputé couvrir, d'une part, les risques de prévision ou d'accidents conjoncturels et, d'autre part, le profit que doit dégager l'opération.

Lorsque l'on passe de cette configuration, celle d'une entreprise du marché concurrentiel, à celle d'une politique publique, les choses se compliquent. Certes, on peut, en premier lieu, prendre en compte les dimensions financières de la décision, c'est à dire procéder exactement à la même évaluation que dans le cas précédent, et donc à l'estimation du TRI du projet. Le calcul sera exactement le même que dans le cas d'un opérateur privé qui serait en charge de la réalisation de l'infrastructure et de son exploitation, mais son résultat ne sera pas utilisé de la même façon pour inspirer la décision. Là encore, nous pouvons aller du plus simple au plus complexe.

Dans l'hypothèse la plus simple, on peut supposer que l'opérateur public soit soumis à une contrainte d'équilibre financier de ses opérations et qu'il ne puisse bénéficier de financement public. Il doit alors se limiter aux opérations susceptibles d'être gagées sur les recettes à venir. C'est ainsi que s'il peut lever un emprunt de long terme à un taux réel de l'ordre de $4 \%$, il devra attendre de l'opération une rentabilité qui couvre ce taux augmenté d'une marge de sécurité liée aux risques d'erreur de prévision ou d'incident conjoncturel. L'opérateur public n'ayant pas d'obligation de profit, la barre devrait se situer en deçà des $12 \%$ évoqués ci-dessus dans le cas de l'entreprise privée. C'est ainsi qu'à la veille de la réforme de 1997, alors que la SNCF assurait la maitrise d'ouvrage des investissements d'infrastructures, le TRI était considéré comme ne devant pas être inférieur à $8 \%$. 
Dans une hypothèse plus ouverte, qui correspond à beaucoup de situations concrètes, un écart peut apparaître entre le TRI d'un projet et ce TRI plancher en deçà duquel l'opérateur public ne peut engager sa réalisation. Sur la base de critères strictement financiers, un tel projet devrait être récusé. Cependant, si la puissance publique a des raisons d'une autre nature d'en assurer la réalisation, elle peut décider de financer " la différence ", c'est à dire d'apporter une part de financement telle que, pour l'opérateur, la rentabilité attendue du projet soit relevée au niveau du TRI plancher.

Nous reviendrons sur la relation entre le TRI d'un projet et la contribution publique qui est requise. Observons, pour l'instant, que cette sollicitation du contribuable n'est théoriquement justifiée que par des avantages externes au bilan financier. Cela, bien entendu, ne signifie pas qu'ils ne soient pas passibles d'un calcul économique.

En effet, si le bilan actualisé d'un projet est élargi à l'ensemble des coûts et avantages pour la collectivité, y compris divers effets externes, un taux de rentabilité interne peut être calculé. Il est généralement appelé taux de rentabilité socio-économique ou, plus simplement, économique (TRE). Ce n'est plus alors le seul point de vue du transporteur et de son compte de résultat qui est retenu, mais celui de la collectivité tout entière. Les pertes et avantages de tous les agents économiques sont ainsi évalués, comme par exemple, les pertes de recette nettes des modes concurrents ou les variations de surplus des usagers, ou encore les conséquences du projet sur la sécurité ou l'environnement.

Pour les éléments non marchands de ce bilan, le problème se pose de leur équivalent monétaire et doit être résolu, soit par des valorisations révélées par le comportement des usagers, telle la "valeur du temps", soit par des valorisations tutélaires, telle la "valeur du mort ». Comme l'on s'éloigne d'un système ordinaire de prix directement signalé par le marché, il s'agit d'un problème d'autant plus controversé que ses solutions ne sont pas neutres : les investissements de sécurité routière se trouvent favorisés par une valeur tutélaire plus élevée du mort évité ; les investissements ferroviaires par des valeurs accrues des pollutions atmosphériques ; etc. Afin de dépasser ces controverses, des prescriptions visant à harmoniser les évaluations entre les modes de transports, en particulier socio-économiques, ont été mises à jour par un groupe du Plan présidé par Marcel Boiteux (Commissariat au Plan, 1996). Les « évaluateurs " sont ainsi réputés se soumettre à ces prescriptions ${ }^{4}$.

La puissance publique, confrontée à une masse de projets d'infrastructures qui dépasse de beaucoup ses capacités de financement, est donc instruite de quelques éléments synthétiques qui lui permettent, en principe, d'optimiser le rendement social de ses arbitrages : pour chaque projet, une estimation du TRI, dont dérive un éventuel besoin de finance- 
ment public est complétée par une estimation du TRE dont la valeur peut justifier cette contribution publique.

Un double choix doit alors être fait. Il y a celui, bien sûr, des projets que l'on décide de réaliser. L'usage et le souci de créer plus de richesse que l'on en détruit ont voulu que soient, en principe, retenus prioritairement les projets présentant le TRE le plus élevé parmi les projets dont le bilan socio-économique apparaît positif. Ce bilan est établi avec "le taux d'actualisation du Plan » actuellement fixé à $8 \%$. Cela signifie que tout projet dont le TRE est supérieur à ce seuil, et qui a donc une VAN positive, est réputé rentable pour la collectivité et donc appelé à être réalisé, mais après que soient achevés les projets plus rentables encore'.

Mais si l'on fait, un instant, abstraction des inerties institutionnelles, il y a aussi à faire un deuxième choix qui est celui de la nature du maître d'ouvrage. Ce peut être l'Etat, comme pour les routes ou autoroutes non concédées ; ce peut être un établissement public, comme pour les lignes nouvelles à grande vitesse; ce peut être un opérateur privé, comme pour l'Eurotunnel ou Orlyval à son origine. Indépendamment de la tyrannie du statu quo, cet arbitrage-là n'est pas aussi simple que le suggèrent certains des chiffres que nous avons cités.

\section{DU PUBLIC AU PRIVÉ}

En effet l'évocation des ordres de grandeur des TRI exigibles par une entreprise publique et par une entreprise privée, $8 \%$ pour l'une et $12 \%$ pour l'autre, nous suggère que le recours à la première sera moins coûteux pour les finances publiques pour toute opération dont le TRI serait inférieur à $12 \%$. Si nous nous en tenons à ces ordres de grandeur, la situation peut être schématisée sur la figure 1 ci-après où apparaissent, selon la valeur du TRI du projet considéré, les zones pour lesquelles une subvention sera nécessaire.

Si l'on s'en tient à ces distinctions, seuls les projets dont le TRI est supérieur à $12 \%$, peuvent être confiés indifféremment à un opérateur public ou privé. Encore que la puissance publique puisse souhaiter que la part de la rentabilité du projet qui correspond à un profit vienne abonder le résultat comptable de l'opérateur public, que ce soit pour couvrir d'autres activités déficitaires ou pour accroître ses capacités de financement.

En-deçà de ce taux exigible dans le secteur concurrentiel, le recours à un opérateur public apparaît toujours moins coûteux pour les finances publiques. D'une certaine manière cette différence explique le retrait progressif des compagnies privées de la scène du transport ferroviaire. Alors que celles-ci, adossées à un puissant soutien bancaire, devaient drainer le financement privé qui allait assurer un développement im- 


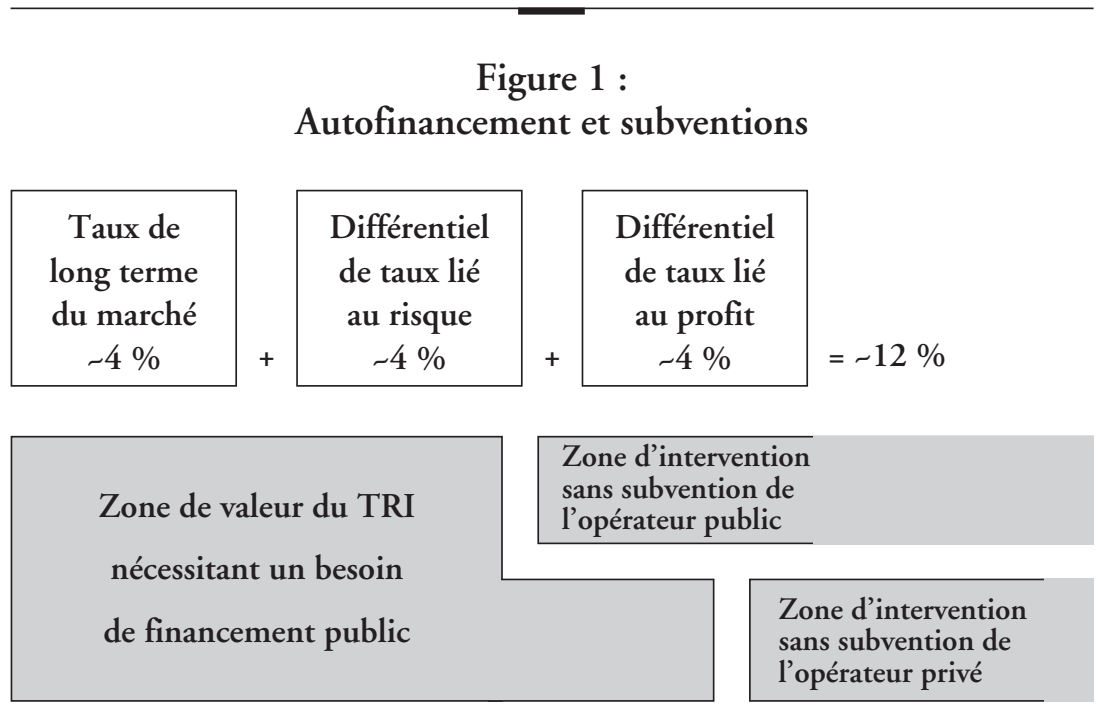

Plage de variation du taux de rentabilité interne

pressionnant de notre réseau ferroviaire à partir de 1832 et avec une accélération foudroyante ${ }^{6}$ au cours du Second Empire, l'intervention financière de l'Etat s'est ensuite imposée, à mesure que la rentabilité financière des nouvelles lignes s'abaissait. C'est ainsi que le plan Freycinet de 1878, appuyé par Gambetta et dont la vocation officielle était de coordonner les constructions et d'assurer ainsi la cohérence de la morphologie des réseaux, consacre également l'intervention des finances publiques. L'adhésion des compagnies à ce plan fut concrétisée par la conventions de 1883 (conventions Raynal). Les grandes compagnies rachetèrent ainsi les concessions faites à de plus petites, mais en obtenant chaque fois de l'Etat de nouveaux avantages financiers. En 1908, le réseau de l'Ouest, déficitaire, devait même être incorporé à l'Etat.

Tout s'est donc passé comme si les investissements successifs du réseau ferré s'étaient progressivement déplacés de la droite vers la gauche sur notre schéma. Avant même l'apparition de ce redoutable concurrent que sera le véhicule automobile, une logique de rentabilité décroissante du capital va apparaître, qui est intimement liée à la hiérarchie des corridors et voies de communication : si les axes les plus importants qui seront irrigués par ces nouveaux chemins sont appelés à être très fréquentés et si les premiers compléments ou prolongements qui leur sont apportés dégagent, à la faveur d'un effet de réseau, une rentabilité encore très honorable, il vient un moment où le segment supplémentaire coûte plus cher qu'il ne rapportera jamais. S'il est justifié par des avantages collectifs non marchands, c'est au contribuable de couvrir ce que le consentement à payer des usagers ne suffit pas à financer. 
Il appartient alors à la puissance publique de maîtriser cette contribution. D'une part en tenant compte de la saturation du territoire par un réseau dont l'extension est à rendement décroissant, ce que devrait théoriquement lui confirmer un calcul de rentabilité socio-économique propre à délimiter le domaine de pertinence d'un réseau, par exemple autoroutier ou ferroviaire. D'autre part en substituant progressivement à l'opérateur privé un opérateur public réputé moins exigeant en rentabilité financière, ce qui devrait théoriquement limiter la contribution publique. L'épilogue de cette évolution a été, pour le secteur ferroviaire, la nationalisation du système avec la création, le 1er janvier 1938, de la Société nationale des chemins de fer français, dont l'Etat détenait $51 \%$ du capital social, les $49 \%$ restants revenant aux anciennes compagnies réduites au rôle de sociétés de portefeuille. A l'expiration du contrat, au $1^{\text {er }}$ janvier 1983, l'Etat devait devenir seul propriétaire et exploitant ${ }^{7}$.

Un scénario analogue a pu être observé dans la plupart des pays, seuls les Etats-Unis se singularisant en conservant le régime des compagnies (plus de 400 existaient encore au début des années 60). Pratiquement, toutes les nations européennes ont créé des réseaux d'Etat ou nationalisé les anciens réseaux. Le chemin de fer a ainsi rejoint, d'une certaine manière, la logique des réseaux routiers.

L'idée d'un recours à un opérateur privé, maître d'ouvrage et concessionnaire, a pourtant refait son chemin dans la période récente, donnant même lieu à de multiples expériences concrètes. Outre des expériences notables dans pratiquement toutes les sortes d'infrastructures de transport, en particulier dans les domaines aéroportuaires et portuaires, le cas français des autoroutes concédées est l'un des plus significatifs. Le décret du 12 mai 1970 a, en effet, ouvert la possibilité d'accorder concession et garantie à des sociétés à capitaux entièrement privés. Il en est résulté la création de quatre sociétés privées qui s'ajoutaient aux cinq sociétés d'économie mixte créées entre 1955 et 1963. La réduction progressive des garanties ou des avances de l'Etat, ajoutée aux difficultés de trésorerie liées à la crise du début des années 80 , devait conduire à des situations déficitaires de trois des quatre sociétés privées et à leur transformation en sociétés d'économie mixte ${ }^{8}$.

En dépit de ces difficultés financières, comme de celles de l'Eurotunnel ou d'Orlyval, l'hypothèse du recours à des opérateurs privés a été de nouveau ouverte, en particulier dans le monde anglo-saxon. C'est ainsi qu'un auteur comme Gordon Mills [1996], qui reconnaît qu'un surcroît de financement public est théoriquement nécessaire avec un opérateur privé, relève de multiples raisons qui peuvent justifier néanmoins ce recours. On peut les regrouper en trois séries de justifications.

La première tient à la possibilité, pour un opérateur privé, de mieux gérer la construction et le fonctionnement du projet considéré. Cela 
revient à supposer que le TRI propre au projet n'est pas le même selon que la gestion est assurée par une administration ou un établissement public ou par une entreprise théoriquement familiarisée avec les efforts permanents d'optimisation. De multiples explications de cette différence sont évoquées : des salaires moins élevés dans le secteur privé pour certaines catégories de personnels, une plus grande flexibilité, des délais de construction plus rapides qui hâtent le retour sur investissement ou, encore, une plus grande capacité à résister à des demandes politiques impliquant des coûts supplémentaires.

La seconde série de justifications est particulièrement importante dans les pays peu accoutumés aux infrastructures à péage. J.A. Gomez-Ibanez et J.R. Meyer [1993] font observer qu'un péage est mal acceptélorsque l'Etat est propriétaire de l'infrastructure alors que cela paraît naturel lorsque l'ouvrage est financé par une entreprise privée. Cette solution est ainsi souvent la seule qui permette d'appliquer le principe de l'usager-payeur.

La troisième série de justifications du recours à un financement privé tient à l'excès d'endettement public, qu'il concerne l'opérateur public susceptible d'assumer le projet ou l'Etat lui-même. Même s'il est gagé sur des recettes à venir, l'endettement supplémentaire peut avoir des inconvénients en matière de cotation financière du premier ou, plus généralement en regard d'un objectif de maîtrise de la dette publique?.

Ces séries de raisons, qui peuvent évidemment se conjuguer, peuvent ne pas suffire à compenser le surcoût éventuel pour les finances publiques de la solution privée, ainsi que le reconnaît G. Mills. Il rejoint ainsi beaucoup d'auteurs qui récusent un choix systématique pour l'une ou l'autre des solutions et suggèrent plutôt de déterminer au cas par cas la solution la mieux appropriée aux circonstances.

\section{LE PARADOXE DES EFFETS DU TRI}

Retenons de ce qui précède que la rentabilité des nouvelles infrastructures s'affaisse à mesure que sont exploités les meilleurs gisements de rentabilité et que, dès lors, le financement de projets qui présentent tout de même un TRE élevé est d'autant plus problématique que l'effort de maîtrise de la dépense publique est aujourd'hui généralisé. La question qui surplombe toutes les autres est alors de savoir comment assurer le développement des réseaux le plus efficace sous cette contrainte de rareté devenue plus active. Il n'est pas dit que l'arbitrage entre financement public et financement privé soit alors plus décisif que l'ordre dans lequel les investissements sont programmés.

Plutôt que d'établir et d'analyser la relation relativement complexe qui peut exister entre le taux de rentabilité interne d'un projet et le montant de subvention requis pour que son financement soit assuré, examinons une situation singulière sur laquelle nous pouvons induire 
quelques résultats de portée générale. Considérons, en effet, un projet d'investissement dont la silhouette chronologique des coûts et bénéfices est relativement classique, à savoir des coûts d'investissements concentrés dans les années qui précèdent la mise en service du projet et des bénéfices initiaux relativement modestes mais croissants dans la durée.

Pour fixer les idées, nous supposons que le projet considéré représente un investissement de 2,3 milliards de francs et que sa rentabilité financière correspond à un TRI de 4,24\%. Nous supposons également, pour l'instant, qu'un opérateur unique a la charge du financement et de la maitrise d'ouvrage, ainsi que de l'exploitation commerciale après la mise en service. Il s'agit donc d'une situation comparable à celle d'une société d'autoroute dans le système français ou encore à celle de la SNCF avant la réforme de 1997. Le graphique ci-dessous, issu de simulations du modèle CALCECO [B. Faivre d'Arcier, D. Mignot, 1998], nous livre le montant de subvention qu'il faudra apporter à l'opérateur pour lui assurer le taux de rendement interne qui figure en abscisse. Nous l'appelons ainsi pour le distinguer du taux de rentabilité interne inhérent au projet. Pour la commodité de la lecture, le pourcentage de la subvention par rapport au coût est également reporté sur la courbe avec un pas de $5 \%$.

Ce cas très particulier n'est pas très éloigné de nombre de situations réelles, à un coefficient multiplicatif du coût et des subventions près. Quelques projets inscrits dans les schémas nationaux en vigueur (et dont le TRE est au moins égal à $8 \%$ ) présentent, en effet, des taux de rentabilité interne voisins de $4 \%$ et une configuration chronologique des coûts et des bénéfices proche de celle qui est retenue dans cette simulation. Une telle chronique, en tout état de cause, détermine la silhouette de la courbe cidessus, dès lors que les coûts et bénéfices sont soumis à un calcul d'actualisation. Or, cette silhouette, à son tour, peut être déterminante pour la rationalité économique et financière des acteurs.

Il est bien normal que le besoin de subvention soit une fonction croissante du taux de rendement interne qu'il s'agit d'assurer à l'opérateur. La pente de la courbe est, en outre, fortement décroissante, ce qui est plus contre-intuitif. Cela signifie, en particulier, que les premiers écarts entre le TRI visé et le TRI de l'opération sont très coûteux : un écart de deux points entre ces deux valeurs implique un besoin de financement public du projet qui représente $45 \%$ de son coût.

Cette première observation nous suggère que l'effet de levier $d u$ financement public sur le rythme des investissements sera d'autant plus puissant que seront choisis prioritairement les projets, s'ils existent, dont le TRI est très proche du TRI visé. Le choix d'un opérateur privé serait, dans ce cas, très coûteux à moins que le TRI de l'opération soit significativement relevé par une efficacité sensiblement supérieure de la gestion privée par rapport à celle d'un établissement public. 
Figure $n^{\circ} 2$ :

Variation du besoin de subvention selon le TRI visé

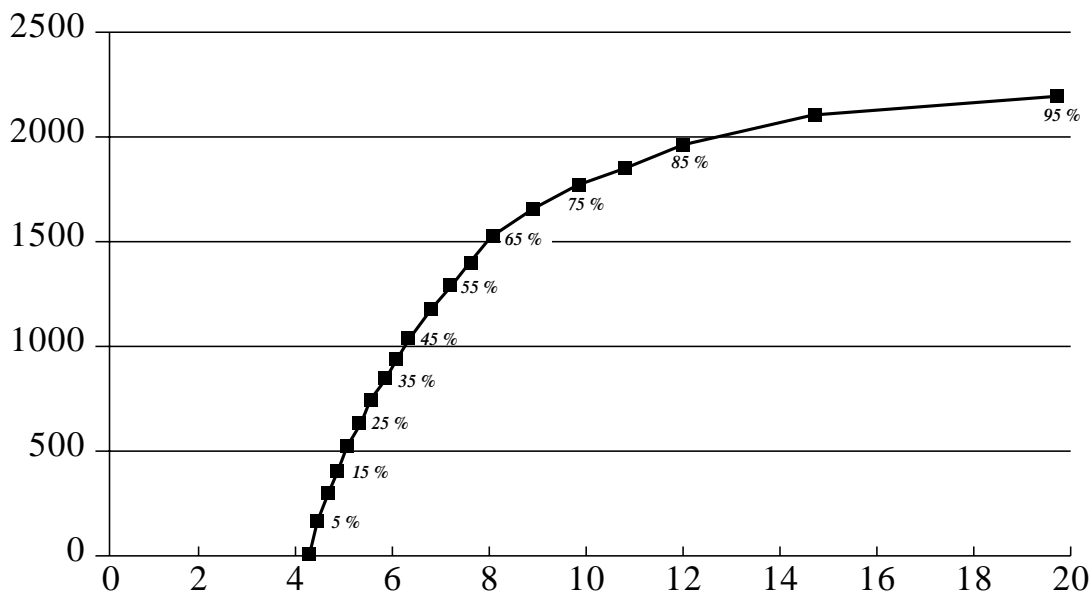

Il y a cependant une deuxième conséquence à la forme de la courbe et en particulier à sa pente décroissante. En effet, plus le TRI de l'opération est faible par rapport au TRI visé, plus le coût marginal pour les finances publiques d'un accroissement de ce TRI visé est faible : c'est ainsi que dans la simulation présentée, le passage de 12 à $14 \%$ du TRI visé représente un accroissement de la contribution publique de moins de $4 \%$ du coût du projet.

On aboutit donc à cet étonnant paradoxe selon lequel le différentiel de coût pour la collectivité qui recourt à un opérateur privé s'estompe pour les projets de faible rentabilité financière.

Ainsi, sous la réserve d'une faible différence entre les efficacités respectives des deux types d'opérateurs, nous pouvons conclure que le recours à un acteur public se justifie pour les projets à forte rentabilité financière, alors que le recours à un acteur privé peut avoir des avantages qui ne seraient pas amputés par des subventions accrues pour des projets de faible rentabilité financière. Ce paradoxe de la rentabilité financière peut expliquer le regain du thème du financement privé à un moment où, précisément, restent à construire des infrastructures qui ont des rentabilités financières moins attrayantes que par le passé. 


\section{BIBLIOGRAPHIE}

Bonnafous A., Crozet Y. (1997) «Evaluation, dévaluation ou réévaluation des lignes à grande vitesse? » Les Cahiers Scientifiques du Transport, N³2.

Cohen Y. (1991) "California's Private Infrastructure Initiative », Journal of Transport Economics and Policy 25

Commissariat Général au Plan (1996) "Transports : pour un meilleur choix des investissements ", Rapport du groupe présidé par Marcel Boiteux, Documentation Française.

Commissariat GÉnÉral au Plan (1997) «Transports : le prix d'une stratégie », Rapport de l'atelier présidé par Alain Bonnafous, 2 vol., Documentation Française.

Faivre d’Arcier B., Mignot D. (1998) «Using Economic Calculation as a simulation Tool to Assess Transport Investments ", Communication à la $8^{\text {ème }}$ WCTR, Anvers.

Gomez-Ibanez J.A., Meyer J.R. (1993) Going Private, Brooking Institution, Washington D.C.

Mills G. (1996) Public Infrastructure : «Private Ownership or Contracting Out », Working Paper $\mathrm{N}^{\circ} 18$, Center for Microeconomic Policy Analysis, University of Sydney.

\section{NOTES}

1. En cherchant bien on trouve pourtant dans la période récente des "décisions " représentant quelques dizaines de milliards de dépenses publiques, et qui dérogent pourtant à au moins une de ces conditions.

2. Pour être complet, nous devrions également évoquer l'instrument réglementaire. Mais, outre que l'expression de "pentagone magique " n'est pas heureuse, nous touchons là à une dimension du problème qui n'est pas centrale dans l'analyse que nous proposons.

3. L'usage veut que les bilans actualisés soient établis en " francs constants " afin de ne pas ajouter aux incertitudes celle qui pèserait sur les prévisions d'inflation à long terme. Contrairement à une idée répandue, cela n'implique pas une hypothèse de prix constants et n'exclut pas que des hypothèses puissent être introduites sur des évolutions prévisibles de certains prix relatifs.

4. Celles-ci sont, bien entendu, appelées à être précisées ou révisées à mesure que le permettront les progrès de la connaissance relative aux valeurs révélées et aux externalités.

5. Ce "seuil », ou taux d'actualisation du Plan, a été ramené de $10 \%$ à $8 \%$ au début des années 80 et n'a pas été révisé depuis. Cela a eu évidemment pour effet d'allonger sensiblement la liste de projets réputés rentables pour la collectivité. Mais cela n'a pas empêché que le poids des dépenses d'infrastructure dans le PIB soit, dans le même temps, diminué [Commissariat général du Plan, 1993]. Il eut été cohérent avec la contrainte de financement de ne pas l'abaisser ou même de le relever. Relevons également que le $8 \%$ du Plan est un seuil de TRE alors que le $8 \%$ garanti à la SNCF est un TRI et que l'égalité entre ces valeurs est fortuite.

6. Le terme n'est pas excessif si l'on songe que l'on a ouvert, dans cette période, jusqu'à un millier de kilomètres de lignes nouvelles par an, ce qui est à rapprocher de la quinzaine d'années aujourd'hui nécessaires pour mettre en service une longueur du même ordre de lignes nouvelles à grande vitesse.

7. Cette échéance explique la date du 30 décembre 1982 de la promulgation de la Loi d'orientation de transports intérieurs (LOTI), qui fait de la SNCF un établissement public industriel et commercial qui reçoit en dotation les biens immobiliers et mobiliers antérieurement concédés à la société anonyme.

8. Ainsi ne demeure aujourd'hui, parmi les 9 sociétés concessionnaires, qu'une société privée, la Compagnie financière et industrielle des autoroutes (COFIROUTE).

9. Ainsi en est-il des pays de l'Union Economique et Monétaire qui doivent respecter le critère de convergence relatif à la dette (au plus $60 \%$ du PIB). Indépendamment de ce cas particulier, tout pays dont la dette publique est élevée peut souhaiter se libérer de "l'effet boule de neige " par lequel la charge de la dette vient abonder le poids de la dette dès lors que les taux d'intérêt sont supérieurs au taux de croissance nominale. 